\title{
Mismatch repair status and PD-L1 expression in clear cell carcinomas of the ovary and endometrium
}

\author{
Brian C Willis, Emily A Sloan, Kristen A Atkins, Mark H Stoler and Anne M Mills \\ University of Virginia Department of Pathology, University of Virginia, Charlottesville, VA, USA
}

\begin{abstract}
Clear cell carcinoma represents a distinct histologic type of müllerian carcinoma that is resistant to conventional chemotherapy. Expression of programmed cell death ligand (PD-L1) has been associated with immune evasion in numerous tumor types and may be used to identify patients who will benefit from targeted immunotherapy, particularly in the setting of mismatch repair defects. We evaluated PD-L1 expression in 23 ovarian clear cell carcinomas and 21 endometrial clear cell carcinomas, and correlated expression with mismatch repair status. Tumor PD-L1 staining was seen in $43 \%$ of ovarian tumors and $76 \%$ of endometrial tumors, including $71 \%$ of cases (67\% of ovarian and $75 \%$ of endometrial) with mismatch repair defects. Extensive tumoral staining ( $>50 \%$ ) was seen in only one case (an endometrial case with MSH6 loss). However, tumoral PD-L1 expression remained common in mismatch repair-intact tumors and mismatch repair status was not significantly correlated with PDL1 expression. The increased incidence of PD-L1 positivity in tumor cells $(P=0.04)$ in endometrial vs ovarian clear cell carcinomas suggests differences in the tumor microenvironment of these histologically and molecularly similar tumors that may inform treatment options. These results suggest that clear cell histology may be a useful susceptibility marker for immunotherapy targeting the PD-1/PD-L1 axis irrespective of mismatch repair status, particularly in endometrial carcinomas.

Modern Pathology (2017) 30, 1622-1632; doi:10.1038/modpathol.2017.67; published online 28 July 2017
\end{abstract}

Clear cell carcinoma of müllerian origin is a rare and distinct histologic subtype, which occurs in the ovary, uterus, cervix, and vagina. Histologically, it is characterized by polygonal to hobnail-shaped cells with clear to eosinophilic cytoplasm and at least focal high-grade nuclear atypia. These cells can be arranged in papillary, solid, or tubulocystic patterns. ${ }^{1}$ Ovarian clear cell carcinomas have an unfavorable stage-matched outcome compared to other subtypes of epithelial ovarian cancer ${ }^{2,3}$ and are particularly resistant to platinum-based chemotherapy. ${ }^{4,5}$ Relatively limited data are available regarding the prognostic implications and chemosensitivity of endometrial clear cell carcinoma, but they are commonly associated with aggressive clinical behavior with a high incidence of extrauterine spread and occult metastases even when the tumor is apparently confined to the uterus. ${ }^{6}$

Correspondence: Dr A Mills, MD, University of Virginia Department of Pathology, University of Virginia, 1215 Lee Street HEP 3rd Floor, Room 3001, Charlottesville, VA 22908, USA.

E-mail: amm7r@virginia.edu

Received 30 March 2017; revised 2 May 2017; accepted 6 May 2017; published online 28 July 2017
The immune system has a key role in modulating tumorigenesis and facilitating tumor clearance in a variety of malignancies. ${ }^{7-9}$ The functionality of the immune system as a tumor-clearing apparatus relies on the balance of immune suppression, to avoid immunopathology, and appropriate immune stimulation, to enhance cytotoxicity against tumor cells. Conversely, unbalanced immune responses or tumor types that induce a response that is favorable to tumor growth via cellular messaging may result in an immune microenvironment that promotes tumor growth and progression. Immune checkpoint pathways are increasingly understood to represent important mechanisms regulating the $\mathrm{T}$ cells involved in antitumor cytotoxicity. One such pathway is mediated by programmed cell death 1 (PD-1), which is expressed chiefly on activated lymphocytes, and its ligand programmed cell death ligand 1 (PD-L1), which is upregulated on antigen-presenting cell types in response to cytokine mediators in a normal immune response. ${ }^{10}$ The interaction of PD-1/ PD-L1 results in immune suppression through inhibition of T-cell proliferation, activation of regulatory $\mathrm{T}$ cells, and induction of T-cell anergy and apoptosis. ${ }^{11,12}$ The normal function of the PD-1/PDL1 pathway is thus essential to quenching immune 
responses that may otherwise become pathologic. However, if the pathway is exploited by malignancy it can interfere with an appropriate host immune response and foster tumor progression. ${ }^{9}$ The PD-1/PD-L1 pathway is of considerable clinical interest due to the success of PD-1 and PD-L1 inhibitor therapy in numerous malignancies, including melanoma, non-small cell lung carcinoma, urothelial carcinoma, and renal cell carcinoma. ${ }^{13-18}$

Tumor cell expression of PD-L1 is one mechanism by which malignant cells may co-opt the PD-1/PD-L1 immune inhibitory pathway. ${ }^{9,16}$ In the gynecologic tract, PD-L1 expression has been demonstrated in tumors of various types in both the ovary and the endometrium, including clear cell carcinoma, and is especially high in endometrial cancers with mismatch repair deficiency/microsatellite instability. ${ }^{19,20}$ Clinical trials from the lung have correlated tumor cell expression of PD-L1 with improved tumor response to PD-1/PD-L1 inhibitor therapy. ${ }^{15,17,21-24}$ In addition, there is interest in the significance of PD-L1 expression by peritumoral and intratumoral immune cells (the so-called 'immune stroma'), which may have a role in immune modulation and tumor evasion of immune attack. ${ }^{15,25,26}$ Tumoral and immune stromal cell expression of PD-L1 has been shown to be higher in malignancies with higher levels of associated inflammation, including triple-negative breast cancers $^{25,27-29}$ and mismatch repair-deficient tumors of both the colon and the endometrium. ${ }^{19,20,30,31}$ Notably, one series from the endometrium showed strong diffuse PD-L1 expression in an endometrial carcinoma with clear cell features. ${ }^{20}$

Data on PD-L1 expression in ovarian carcinoma derive chiefly from serous carcinomas. Some studies of serous carcinoma show PD-L1 primarily restricted to tumor-associated macrophages, while others show instances of positive tumor cell staining, particularly in the setting of BRCA mutations. ${ }^{32-34}$ PD-L1 expression in ovarian clear cell carcinoma has not been well studied. A subset of ovarian clear cell carcinoma is known to occur in the setting of Lynch syndrome or to harbor somatic mutations in mismatch repair genes. ${ }^{35,36}$ Furthermore, ovarian clear cell carcinomas show a higher number of CD3 + tumor-infiltrating lymphocytes compared to their serous counterparts. ${ }^{37}$ These features make the prospect of immune therapy appealing in this tumor type, which is generally resistant to traditional platinum-based chemotherapy.

To better understand the role of PD-L1 in clear cell carcinomas, we herein characterize PD-L1 immunohistochemical expression in the tumor and peritumoral immune compartment of 23 ovarian and 21 endometrial clear cell carcinomas and discuss the therapeutic implications of our findings.

\section{Materials and methods}

Cases were identified by performing a natural language search in Co-Path for internal ovarian and endometrial cases diagnosed as 'clear cell carcinoma' from 1995 to 2016. The entire case was reviewed to confirm the diagnosis and to identify the best tumor-containing slide. Diagnostic confirmation required the identification of uniform morphologic features of clear cell carcinoma, including tubulocystic, papillary, or solid architecture, prominent hyalinization, large tumor cells with at least some hobnail nuclei, and clear cytoplasm. Notably, clear cytoplasm was not required throughout, however areas lacking this feature were still required to retain the other morphologic findings of clear cell carcinoma. Given the controversy regarding the diagnosis of clear cell carcinoma vs mixed carcinoma/endometrioid or serous carcinomas with clear cell features (particularly in the endometrium), only cases with pure clear cell morphology were included for analysis. This was determined based on multiheaded scope review by three gynecologic pathologists (AMM, KAA, and MHS) and one gynecologic pathology fellow (BW). Unanimous agreement by all four pathologists on the diagnosis of pure clear cell carcinoma was required for study inclusion.

PD-L1 immunostaining was performed on whole tissue sections using the SP142 antibody clone (dilution 1:200; Spring Bioscience) on the Leica Bond III platform. This stain has been previously validated in our laboratory on a large array of nonsmall-cell lung carcinomas against results obtained on the Dako 22C3 antibody using the Dako platform with satisfactory results (eg, no discrepant cases at clinically relevant thresholds). Placental tissue was used as a positive control, with circumferential villous staining without significant background stromal staining required for validity. PD-L1 immunohistochemistry staining was scored in both the tumor and peritumoral immune compartment (also the known as the 'immune stroma'). Normal liver and kidney tissue cores were also used as controls.

Tumor cell expression was considered positive when $\geq 1 \%$ of cells showed circumferential membranous staining. The proportion of positive cells was further categorized in strata of $1-5 \%, 6-10 \%$, $11-25 \%, 26-50 \%$, and $>50 \%$, in accordance with cut points that have previously been found relevant in clinical trials for immune checkpoint inhibitor therapy. ${ }^{15,17,23,24}$ Greater than 50\% tumor staining was considered 'extensive.' Staining in the peritumoral immune compartment was considered positive if membranous or cytoplasmic staining was seen in lymphocytes or macrophages in association with the tumor. Because normal control tissues show occasional scattered PD-L1-positive immune cells, $>5 \%$ was selected for as the threshold for immune stromal positivity. Positive cases were further categorized in strata of $5-10 \%, 10-50 \%$, and $>50 \%$. 
Mismatch repair deficiency was determined by the complete absence of nuclear tumor immunostaining for any of the four mismatch repair proteins (MSH2 (clone 25D12, predilute; Leica), MSH6 (clone 44 Mab, predilute; Cell Marque), MLH1 (clone ES05, predilute; Leica Biosystems), and PMS2 (clone MRG-28Mab, predilute; Cell Marque) in the setting of intact control stromal/lymphocyte staining. Cases identified thorough the universal LS screening program (initiated at our institution in 2012) were stained for all four proteins, and cases with dual MLH1/PMS2 loss underwent PCR-based MLH1 hypermethylation testing. Cases stained exclusively for the study were stained with MSH6 and PMS2 initially, and tumors showing loss were further stained for their partner protein (MSH2 and MLH1, respectively). Germline confirmation of Lynch syndrome was not available for any cases.

Statistical analysis was performed using the twotailed Fisher exact test for categorical variables (vassarstats.net). This work was approved by the Institutional Review Board of the University of Virginia.

\section{Results}

\section{Tumoral and Peritumoral Immune PD-L1 Expression in Ovarian Clear Cell Carcinoma}

Some degree of PD-L1 staining, either tumoral or immune stromal, was present in $74 \%(17 / 23)$ of ovarian clear cell carcinomas. Tumoral PD-L1 staining was present in $43 \%(10 / 23)$ of ovarian clear cell carcinomas (Table 1; Figure 1). Considerable intratumoral heterogeneity and patchy staining for PD-L1 was observed across whole sections (Figure 2). All positive ovarian clear cell carcinomas showed tumoral staining in $\leq 25 \%$ of cells; there were no cases with extensive staining.

All ovarian clear cell carcinomas had some degree of peritumoral inflammation, comprised chiefly of lymphocytes and macrophages located in the stroma and luminal spaces immediately adjacent to tumor cells. Peritumoral immune PD-L1 staining was present in $52 \%(12 / 23)$ of ovarian clear cell carcinomas, however no cases showed extensive $(>50 \%)$ immune stromal staining (Table 2; Figure 3). Twenty percent $(2 / 10)$ of cases with tumoral staining were

Table 1 Tumor cell expression of PD-L1 in ovarian (OCCC) and endometrial clear cell carcinomas (ECCC)

\begin{tabular}{lclr}
\multicolumn{2}{l}{ Tumor staining OCCC } & \multicolumn{2}{l}{ Tumor staining ECCC } \\
\hline Negative $(<1 \%)$ & $57 \%(13 / 23)$ & Negative $(<1 \%)$ & $24 \%(5 / 21)$ \\
$1-5 \%$ & $35 \%(8 / 23)$ & $1-5 \%$ & $9 \%(2 / 21)$ \\
$6-10 \%$ & $4 \%(1 / 23)$ & $6-10 \%$ & $33 \%(7 / 21)$ \\
$11-25 \%$ & $4 \%(1 / 23)$ & $11-25 \%$ & $24 \%(5 / 21)$ \\
$26-50 \%$ & $0 \%(0 / 23)$ & $26-50 \%$ & $5 \%(1 / 21)$ \\
$>50 \%$ & $0 \%(0 / 23)$ & $>50 \%$ & $5 \%(1 / 21)$ \\
\hline
\end{tabular}

negative for peritumoral immune PD-L1 staining. Conversely, $33 \%(4 / 12)$ of cases with immune stromal staining were negative for PD-L1 in tumor cells.

A total of three ovarian clear cell carcinomas were known to be associated with endometriosis. PD-L1 expression was not significantly associated with coexistent endometriosis (tumor staining: $P=0.56$; immune stromal staining: $P=0.22$ ) tumor staining.

\section{Tumoral and Peritumoral Immune PD-L1 Expression in Endometrial Clear Cell Carcinoma}

Some degree of PD-L1 staining, either tumoral or immune stromal, was present in 90\% (19/21) of endometrial clear cell carcinomas. Tumoral PD-L1 staining was present in $76 \%(16 / 21)$ endometrial cases (Table 1; Figure 4). Extensive tumor staining $(>50 \%)$ was seen in one case $(4 \%)$. Considerable intratumoral heterogeneity and patchy staining for PD-L1 was observed across whole-section cores (Figure 2).

All endometrial clear cell carcinomas showed associated inflammation, which predominantly consisted of lymphocytes located at the tumor/stromal interface and percolating among the tumor cells. Peritumoral immune PD-L1 staining was seen in $76 \%(16 / 21)$ of endometrial tumors with a large proportion of the cases $(57 \%, 12 / 21)$ showing $>10 \%$ staining in the immune compartment and two cases showing $>50 \%$ immune stromal staining (Table 2; Figure 3). Nineteen percent (3/16) of cases with tumoral staining were negative for peritumoral immune PD-L1 staining while another 19\% (3/16) of cases with immune stromal staining were negative for tumoral PD-L1.

\section{Comparison of PD-L1 Staining Between Endometrial and Ovarian Clear Cell Carcinoma}

Endometrial clear cell carcinomas showed statistically significantly higher numbers of cases with tumor expression $(76 \%$ (16/21) vs $43 \%$ (10/23), $P=0.04)$ compared to ovarian cases. Endometrial clear cell carcinomas also showed a trend toward increased immune stromal staining relative to ovarian cases, but this was not statistically significant (76\% $(16 / 21)$ vs $52 \%(12 / 23), P=0.12)$. The difference between presence or absence of any (eg, tumoral and/or immune) PD-L1 staining in endometrial vs ovarian clear cell carcinoma was also not statistically significant (90\% (19/21) vs 74\% (17/23), $P=0.25)$.

\section{PD-L1 and Mismatch Repair Status}

Thirteen percent $(3 / 23)$ of the ovarian tumors showed loss of mismatch repair proteins: all 3 showed dual loss of MSH2 and MSH6. The average age of ovarian clear cell carcinoma patients with 

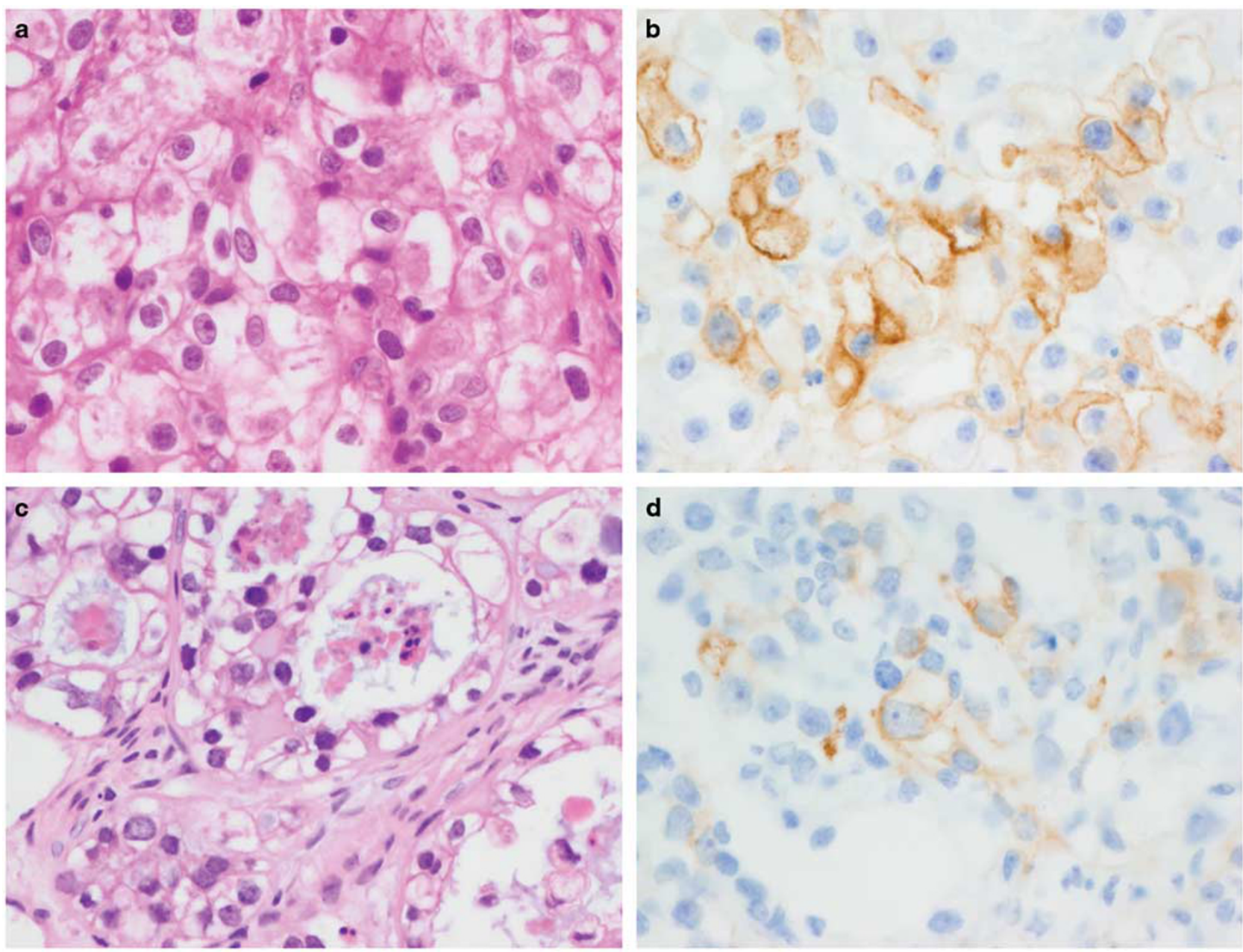

Figure 1 Tumor cell expression of PD-L1 in ovarian clear cell carcinoma. The first case (a, b) shows relatively strong membranous staining in a subset of cells. This case showed loss of MSH2 and MSH6 by immunohistochemistry. The second case (c, d) shows weak staining in scattered cells, which was more typical of ovarian clear cell carcinoma.
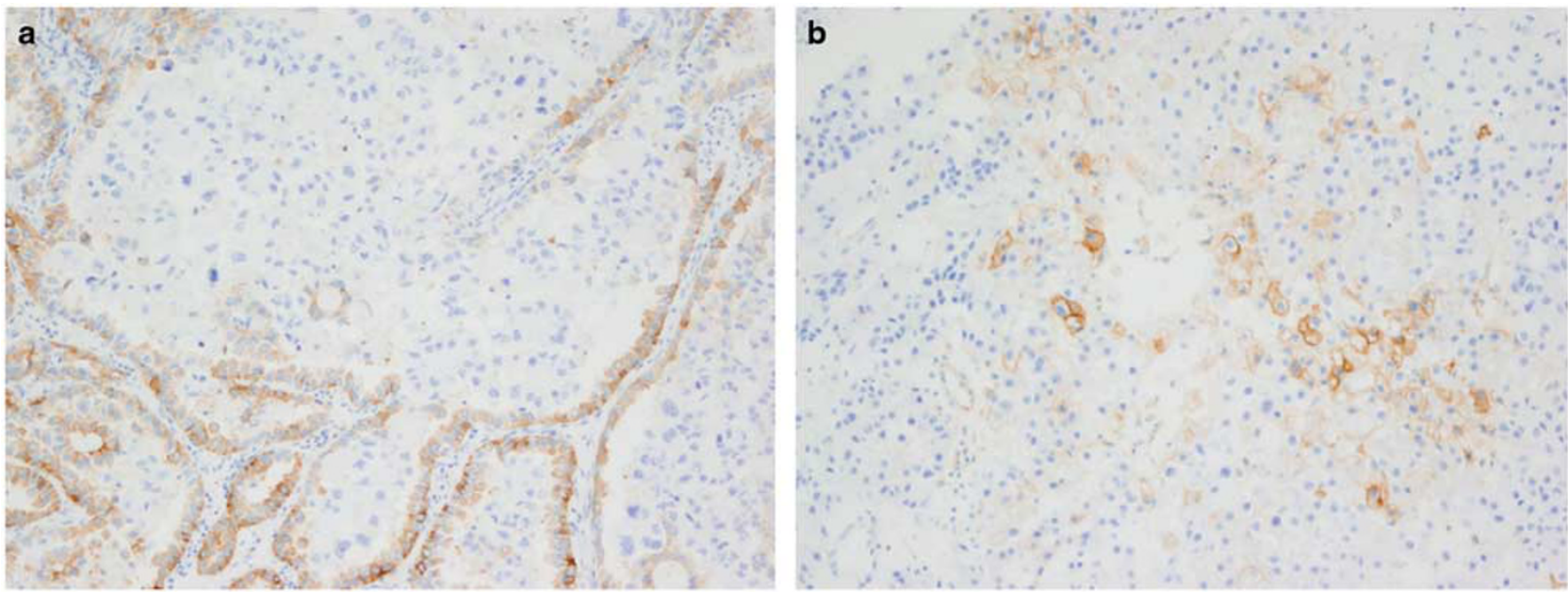

Figure 2 Intratumoral heterogeneity in PD-L1 expression. Many cases from both organ sites showed considerable heterogeneity of PD-L1 staining, demonstrated here in an endometrial (a) and ovarian clear cell carcinoma (b). 
Table 2 Immune stromal expression of PD-L1 in ovarian (OCCC) and endometrial clear cell carcinomas (ECCC)

\begin{tabular}{lclc}
\multicolumn{2}{l}{ Immune staining OCCC } & Immune staining ECCC \\
\hline Negative $(<5 \%)$ & $48 \%(11 / 23)$ & Negative $(<5 \%)$ & $24 \%(5 / 21)$ \\
$5-10 \%$ & $30 \%(7 / 23)$ & $5-10 \%$ & $19 \%(4 / 21)$ \\
$10-50 \%$ & $22 \%(5 / 23)$ & $10-50 \%$ & $48 \%(10 / 21)$ \\
$>50 \%$ & $0 \%(0 / 23)$ & $>50 \%$ & $9 \%(2 / 21)$ \\
\hline
\end{tabular}
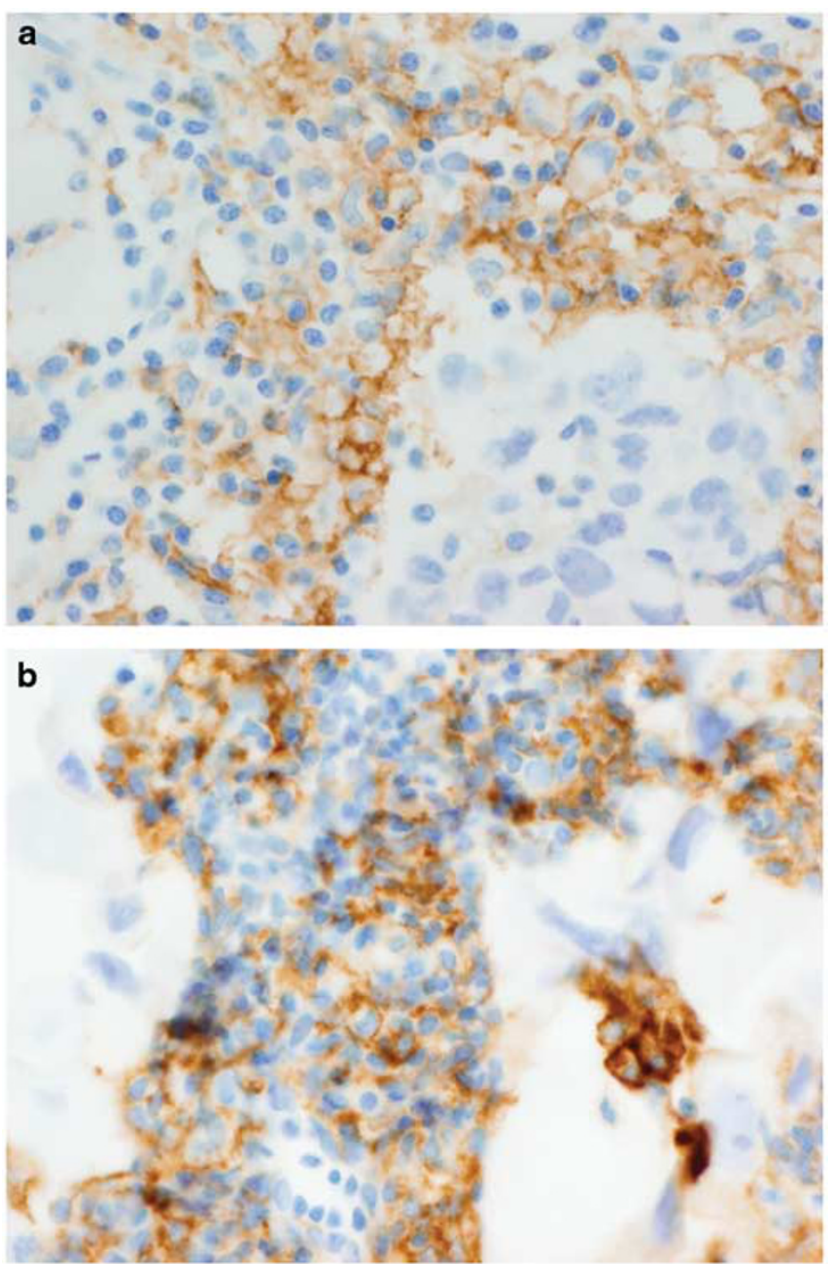

Figure 3 Immune stromal expression of PD-L1. Peritumoral immune cell staining was seen in both ovarian (a) and endometrial clear cell carcinoma (b). Immune stromal cells expressing PD-L1 were chiefly lymphocytes and macrophages.

tumors harboring mismatch repair abnormalities was 60.3 years, compared to 59.4 years for mismatch repair-intact tumors. Sixty-seven percent (2/3) mismatch repair-deficient ovarian clear cell carcinoma showed PD-L1 positivity within tumor cells, including the only ovarian tumors in the series with tumoral PD-L1 positivity reaching 10-25\%. (Table 3). None of the 3 mismatch repair-deficient ovarian cases showed immune stromal staining. Forty percent $(8 / 20)$ of mismatch repair-intact ovarian cases were PD-L1-positive in tumor cells, while $60 \%(12 / 20)$ had immune stromal staining. The relationship between PD-L1 and mismatch repair status was not statistically significant for either tumor $(P=0.55)$ or immune stromal staining $(P=0.09)$.

Nineteen percent (4/21) of endometrial clear cell carcinomas showed mismatch repair deficiencies by immunohistochemistry: one case with loss of MLH1 and PMS2, and PCR-confirmed promoter hypermethylation; one case with isolated loss of MSH6 (Figure 4a and b); and two cases with dual loss of MSH2 and MSH6 (Figure 5). The average age of endometrial carcinoma patients with tumors harboring mismatch repair abnormalities was 60 years, compared to 63 years for mismatch repair-intact tumors. Seventy-five percent (3/4) mismatch repairdeficient tumors were PD-L1-positive within the tumor cells, and $75 \%(3 / 4)$ were positive in the immune cells (Table 3). All four cases showed either tumoral or immune cell staining. The MSH6deficient case showed the strongest tumoral staining out of all the cases in the series with extensive $(>50 \%)$ positivity. The majority of mismatch repairintact endometrial carcinoma cases were also PD-L1positive in tumor cells $(76 \%, 13 / 17)$ and immune stroma $(82 \%, 14 / 17)$, with $88 \%(15 / 17)$ of mismatch repair-intact cases showing tumoral and/or immune cell staining. There was no significant relationship between the presence of PD-L1 expression and mismatch repair status for either tumor $(P=1.0)$ or immune stromal staining $(P=1.0)$.

\section{Discussion}

The success of PD-1 and PD-L1 inhibitors in producing durable clinical responses in a variety of tumor types has led to increased enthusiasm for the use of immunotherapy multiple organ systems, including the gynecologic tract. Emerging evidence shows that high levels of PD-L1 expression can be seen in a subset of mismatch repair-deficient endometrial carcinomas ${ }^{19,20}$ and serous ovarian carcinomas. $^{32-34}$ To date, little is known about PDL1 in clear cell carcinomas of the uterus and ovaries, although data presented in abstract form suggest that high levels of expression can be seen in tumors with this morphology. ${ }^{38}$ Furthermore, the endometrial carcinoma that showed the strongest PD-L1 positivity in the series of mismatch repair-deficient cancers produced by our institution possessed large areas of clear cell morphology. ${ }^{20}$ The association between mismatch repair defects and clear cell morphology is well established in the ovary, ${ }^{39,40}$ and the association between mismatch repair defects and PD-L1 expression has been well established in other locations. ${ }^{30,41,42}$ Finally, müllerian clear cell carcinomas show morphologic overlap with renal clear cell carcinomas (indeed, they were originally termed 'mesonephroid adenocarcinomas') and clear cell tumors of the kidney have shown robust responses to immunotherapy. ${ }^{14,22}$ 

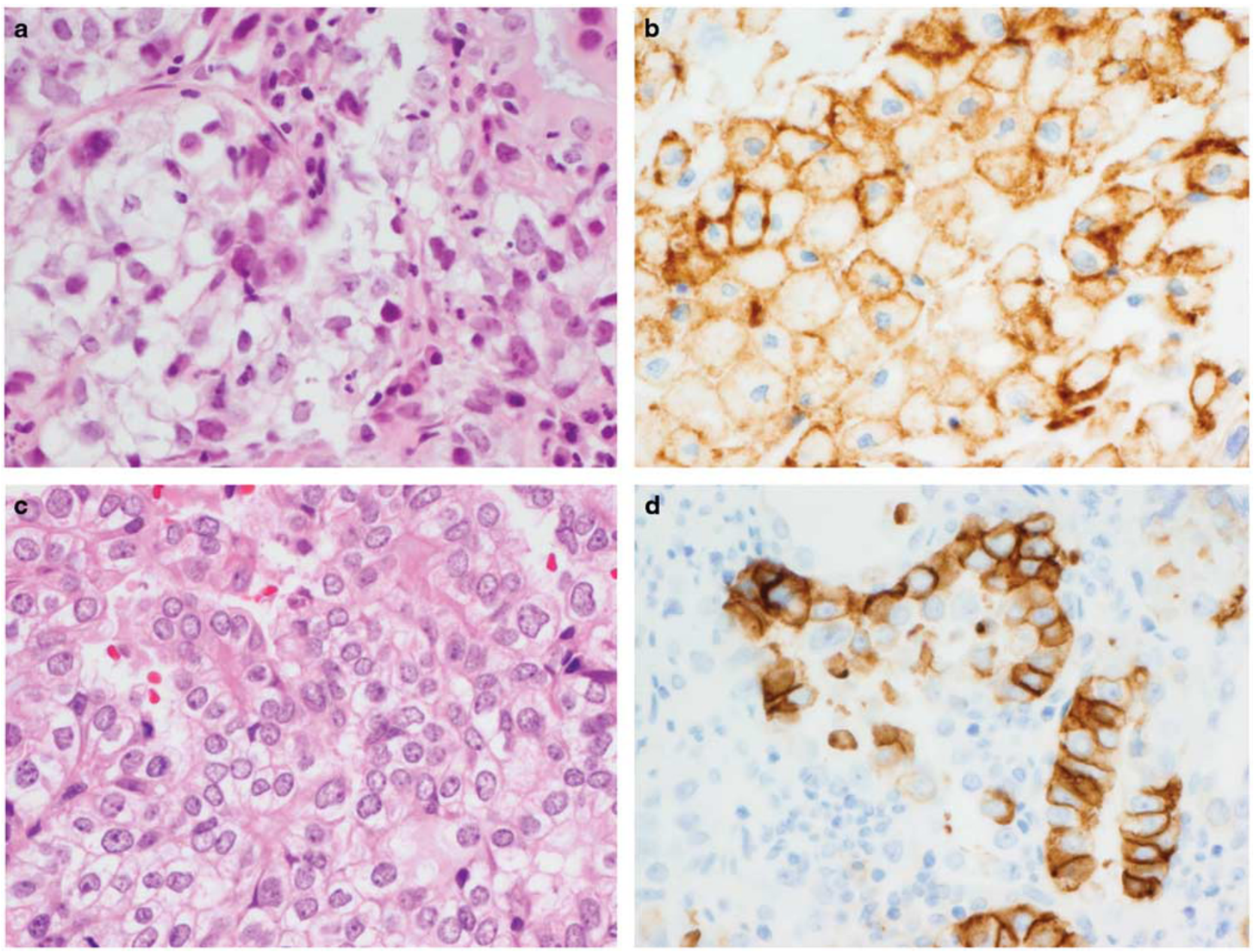

Figure 4 Tumor cell expression of PD-L1 in endometrial clear cell carcinoma. The majority of endometrial clear cell carcinomas showed tumor cell staining with PD-L1, which ranged from diffuse (as evidenced in a and b) to relatively focal (cases c and d). The diffusely positive case in a and b showed loss of MSH6, suggesting an MSH6 mutation.

Table 3 Tumor and immune stromal PD-L1 expression in MMR-deficient ovarian (OCCC) and endometrial clear cell carcinomas (ECCC)

\begin{tabular}{lcll}
\hline MMR-deficient carcinomas & Patient age & Tumor PD-L1 & Immune PD-L1 \\
\hline OCCC, MSH2/6 loss & 65 & Negative $(<1 \%)$ & Negative $(<5 \%)$ \\
OCCC, MSH2/6 loss & 60 & $11-25 \%$ & Negative $(<5 \%)$ \\
OCCC, MSH2/6 loss & 56 & $1-5 \%$ & Negative $(<5 \%)$ \\
ECCC, MLH1/PMS2 loss & 73 & $6-10 \%$ & Negative $(<5 \%)$ \\
ECCC, MSH2/6 loss & 36 & $11-25 \%$ & $10-50 \%$ \\
ECCC, MSH2/6 loss & 77 & Negative $(<1 \%)$ & $5-10 \%$ \\
ECCC, MSH6 loss & 53 & $>50 \%$ & $5-10 \%$ \\
\hline
\end{tabular}

Given these factors, gynecologic clear cell carcinomas may represent particularly good candidates for immunotherapy targeting the PD-1/PD-L1 axis. To our knowledge, this study represents the largest collection of ovarian and endometrial clear cell carcinomas to be examined for PD-L1 expression by immunohistochemistry and highlights potential differences in biology and immunogenicity of these different primary sites. We demonstrate that the majority of endometrial and a subset of ovarian clear cell carcinomas show some degree of tumor cell staining for PD-L1, and that the increased incidence of this finding in endometrial vs ovarian tumors is statistically significant $(76 \%$ vs $43 \%, P=0.04)$. Diffuse $(>50 \%)$ tumor staining is rare, and was here seen in only a single endometrial case and in none of the ovarian tumors. Many endometrial and ovarian clear cell carcinomas also show immune stromal PDL1 positivity and although peritumoral immune staining is more common in endometrial primaries, this is not statistically significant $(76 \%$ vs $52 \%$, $P=0.12$ ). 

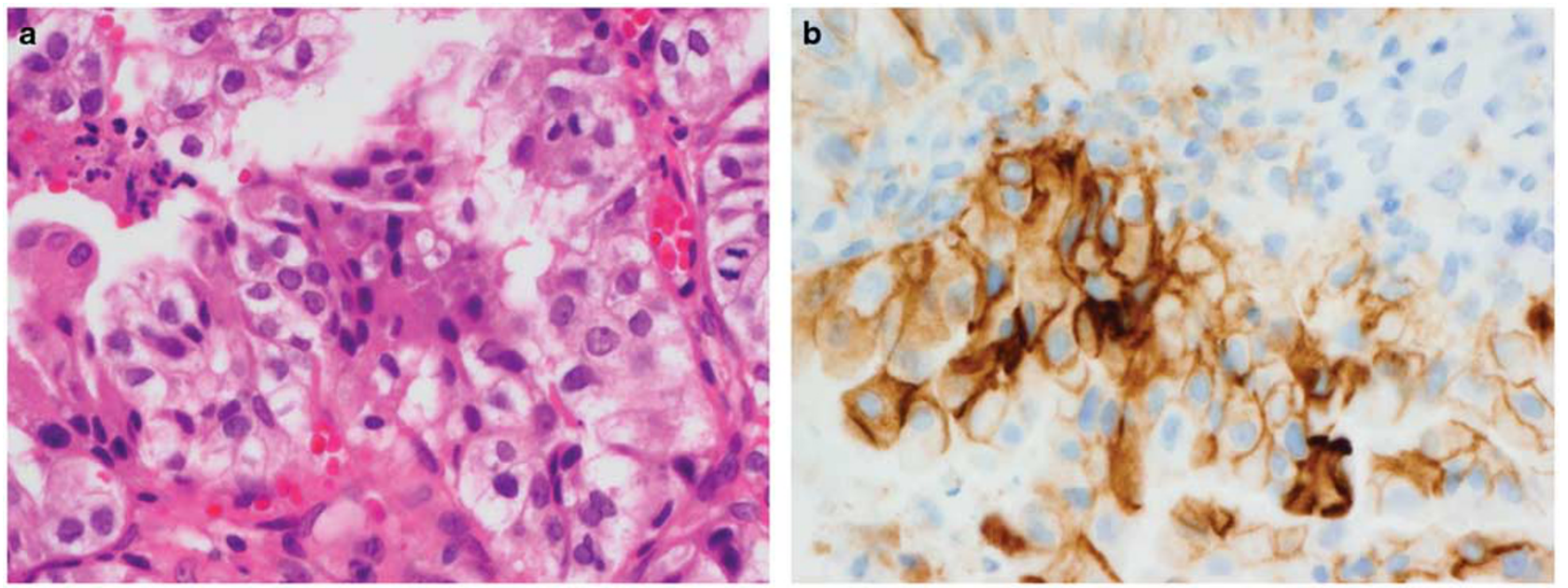

Figure 5 PD-L1 in mismatch repair-deficient tumors. Both endometrial clear cell carcinomas and two of three ovarian clear cell carcinomas with mismatch repair loss by immunohistochemistry showed PD-L1 positivity within tumor cells. This endometrial clear cell carcinoma (a) showed dual loss of MSH2/6 and areas of strong membranous PD-L1 staining (b). (Other mismatch repair-deficient cases are pictured in Figures 1 and 2.)

The differences in tumoral PD-L1 expression between endometrial and ovarian clear cell carcinomas invite questions about the biological underpinnings of these tumors. Importantly, clear cell carcinoma of the endometrium is a less wellunderstood entity than ovarian clear cell carcinoma. This is likely due, in part, to the fact that the diagnosis has often encompassed cases with admixed endometrioid and serous components. In this series, we have excluded morphologically mixed cases and restricted analysis to tumors showing pure clear cell morphology throughout, as unanimously agreed upon by four pathologists with gynecologic pathology training. Indeed, when selecting cases for inclusion in this study 35\% of endometrial cancers originally diagnosed as 'clear cell carcinoma' were excluded based on the presence of endometrioid and/or serous features. The morphologically adjudicated true clear cell cases represented $<1 \%$ of all endometrial carcinomas diagnosed during the 21year study period, in keeping with the rarity of this diagnosis. Common morphologic features that led to reclassification of endometrial carcinoma originally diagnosed as clear cell included prominent areas of secretory differentiation in endometrioid carcinoma and regions of cytoplasmic clearing in serous carcinomas.

Such mixed cases aside, clear cell carcinomas of the ovary and endometrium have been thought of as essentially identical tumor histotypes occurring in different anatomic locations, an assertion that is strengthened by the finding that ovarian clear cell carcinoma often arises from endometriosis. ${ }^{43,44}$ Indeed, retrograde menstruation was long considered a probable source for ovarian clear cell tumors, although more recent thinking suggests tubal origin. Whether they share a common site of origin or not, the morphologic homology of these tumors appears to have a molecular correlate, with gene expression studies showing relatively comparable expression patterns in müllerian clear cell carcinomas across primary locations. ${ }^{45,46}$ Similar mutations in ARID1A and PIK3CA have also been discovered in a subset of ovarian clear cell carcinomas arising in endometriosis and in clear cell carcinomas primary to the endometrium, further attesting to their considerable molecular overlap. ${ }^{43,47}$ Nonetheless, some proteomic differences between ovarian and endometrial clear cell carcinoma have been described, highlighting the need to further investigate the intrinsic differences between these tumors. ${ }^{48}$ Furthermore, while we have attempted to restrict our analysis here to morphologically 'equivalent' cases across locales, it remains possible that ovarian and endometrial clear cell carcinomas represent fundamentally different entities. Differences in the tumor microenvironment may also account for the variation in PD-L1 expression seen in these two locations, as the endometrium and the ovary present different immune milieus. In particular, the endometrial cases in this series more often showed lymphocytic inflammation both at the infiltrating edge of the tumor and intimately admixed with the tumor cells, whereas inflammation associated with ovarian cancers included more macrophages and was less commonly intratumoral. These distinctions in the immune context of these tumors could have implications for PD-1/PD-L1 inhibitor response and warrant further study.

The presence of a subset of endometrial and, to a lesser extent, ovarian clear cell carcinomas showing tumoral and/or peritumoral immune PD-L1 staining is of clinical interest because effective chemotherapeutic regimens are lacking for clear cell carcinoma relative to other histologic subtypes. ${ }^{4,5}$ There is little data available regarding optimal treatment in endometrial clear cell carcinomas, but these tumors often present with extrauterine disease and additional options for adjuvant therapy are desirable. ${ }^{6}$ 
Currently, most of the clinical trial data showing the benefit of PD-1/PD-L1 inhibitors in advanced endometrial cancer relate specifically to tumors with mismatch repair deficiency/high-level microsatellite instability, with one trial using PD-L1 immunostaining in tumor or immune cells of $>1 \%$ as a selection criteria. ${ }^{49,50}$ Tumors with mismatch repair impairments are known to mutate rapidly, resulting in an accumulation of neoantigens that represent potential targets for the host immune system. ${ }^{19,30,31}$ The acquisition of mechanisms of immune evasion, such as PD-L1 expression, is thus of theoretical benefit for mismatch repair-deficient cancers. This is supported by studies showing that PD-L1 expression is increased in mismatch repair-deficient/microsatellite unstable endometrial tumors relative to their mismatch repair-intact/microsatellite stable counterparts. ${ }^{19,20}$ Notably, MSH6 mutations have been particularly associated with increased PD-L1 expression, with 8/8 MSH6-deficient cases in our prior series of mismatch repair-deficient endometrial cancers showing PD-L1 positivity within tumor cells. ${ }^{20}$ In the current clear cell series the case with the highest PD-L1 expression also showed isolated loss of MSH6 by immunohistochemistry. The potential significance of $\mathrm{MSH} 6$ mutations in predicting immunotherapeutic response is further highlighted by a recent report of a patient with advanced endometrioid adenocarcinoma harboring a germline MSH6 mutation who achieved a marked clinical response on the PD-1 inhibitor nivolumab. ${ }^{51}$ That said, the majority of mismatch repair-intact endometrial cancers show PD-L1 expression in our series, suggesting that clear cell histology could serve as a biomarker for PD-1/PD-L1 inhibitor response regardless of mismatch repair status.

Ovarian clear cell carcinoma is also an attractive target for immunotherapy because it is notoriously resistant to traditional platinum-based chemotherapies used in the treatment of other types of ovarian epithelial malignancies. ${ }^{2,4}$ Targeted immunotherapy options have shown some early promise in these tumors. In a recent trial for recurrent platinumresistant ovarian cancer (histologic type unspecified), durable clinical responses were observed in patients with high levels of PD-L1 tumor staining compared to those with lower expression, although these results did not reach statistical significance. ${ }^{52}$ Other trials including three patients with recurrent ovarian clear cell carcinoma have demonstrated sustained response with nivolumab or avelumab therapy. ${ }^{53,54}$ As was the case with endometrial carcinomas, the two mismatch repair-deficient ovarian cases in our series showed PD-L1 tumor cell staining, highlighting the possibility of enhanced vulnerability to immunotherapy in this neoantigenenriched tumor subset of ovarian carcinoma. Importantly, however, the increased incidence of PD-L1 positivity in mismatch repair-deficient ovarian tumors was not statistically significant and a subset of mismatch repair-intact ovarian clear cell carcinoma also showed PD-L1 staining, again suggesting that clear cell morphology could be meaningful for immunotherapeutic targeting irrespective of mismatch repair status. It is worth noting that mismatch repair-deficient ovarian cancers accounted for a slightly higher than expected proportion of cases in this series, with $13 \%$ of ovarian cancers showing mismatch repair protein loss, whereas prior work has estimated the incidence of mismatch repair deficiency in clear cell carcinomas to be $\leq 10 \% .^{55,56}$ This is likely attributable to the small case numbers.

There are important limitations to this study that bear emphasis. Most critically, it is not uniformly evident that the presence and extent of PD-L1 protein expression correlates with PD-1/PD-L1 inhibitor response. ${ }^{21}$ Studies assessing the relationship between tumor PD-L1 expression by immunohistochemistry and response to PD-1/PD-L1 inhibitor therapy have had mixed results across other organ systems. While some studies of melanoma, nonsmall-cell lung cancer, bladder carcinoma, and renal cell carcinoma have linked the presence and/or level of PD-L1 expression with improved response to PD-1 inhibitors, ${ }^{18,57-59}$ other studies in non-small-cell lung cancer and melanoma have shown no significant correlation between PD-L1 staining and antitumor response. ${ }^{17,60}$ Thus, caution must be exercised before equating tumor cell expression patterns with a promise of clinical response, and further studies addressing other markers of possible immunotherapeutic susceptibility (such as neoantigen load) are warranted.

It is likely that the imperfect relationship between PD-L1 expression and clinical response can be attributed at least in part to variability in staining across antibody clones. In this study, PD-L1 expression was interrogated using a single-antibody clone (SP142; Spring Biosciences). We selected the SP142 clone based on the success we have had with the antibody in our clinical lab, its FDA approval in other settings (including treatment of bladder carcinoma and as second-line non-small-cell lung cancer therapy), and the absence of an FDA mandate for a specific clone to be used as a companion diagnostic for any therapies in the gynecologic tract. While this clone has been thoroughly characterized, calibrated, and validated in our hands (including internal validation against the Dako 22C3 clone on the Dako instrument), it is also well established that there is variation in performance between the commercially available antibody clones, ${ }^{61,62}$ and these variations must be considered as limitations in any study assessing PD-L1 by immunohistochemistry.

Despite the shortcomings of immunohistochemistry-based protein expression as a biomarker for PD-L1 susceptibility, barring development of a superior molecular method it is likely to have an ongoing role in rational patient selection for immunotherapy targeting the PD-1/PD-L1 axis, particularly as this treatment becomes more widely administered and more instances of severe side effects are observed. Though generally well-tolerated relative 
to conventional cytotoxic therapies, immunotherapy is not without risks. Significant side effects during and after therapy include myocarditis, acute respiratory distress syndrome, hypophysitis, colitis, and renal failure, and this list is likely to grow as the checkpoint inhibitor therapies are more widely used for longer periods of time. ${ }^{63}$ In addition, these immunotherapies may exclude patients from other more beneficial targeted therapy options such as tyrosine kinase inhibitors due to increased toxicity of combination therapy or a decrease in efficacy of the drug combination. ${ }^{64}$ It is therefore desirable to stratify patients by the likelihood that their tumor will respond optimally to immune checkpoint inhibitor therapy before the initiation of treatment.

In summary, we herein demonstrate that some degree of tumoral and/or immune stromal PD-L1 expression is present in the majority of both ovarian and endometrial clear cell carcinomas, with particularly high expression rates in endometrial cases. The discrepancies in the frequency of tumoral PD-L1 expression between clear cell carcinomas of endometrial and ovarian origin highlight differences between these molecularly and histologically similar tumors, which may relate to their associated microenvironments. While the high levels of PD-L1 staining observed in mismatch repair-deficient cancers underscores the potential utility of immunotherapy in the setting of mismatch repair defects, the finding that many mismatch repair-intact clear cell cancers are also PD-L1-positive suggests that clear cell morphology may be a useful independent marker for targeted immunotherapy.

\section{Disclosure/conflict of interest}

The authors declare no conflict of interest.

\section{References}

1 Kurman RJ. International Agency for Research on Cancer, World Health Organization WHO Classification of Tumours of Female Reproductive Organs, 4th edn. International Agency for Research on Cancer: Lyon, France, 2014, p 307.

2 Mackay HJ, Brady MF, Oza AM, et al. Prognostic relevance of uncommon ovarian histology in women with stage III/IV epithelial ovarian cancer. Int J Gynecol Cancer 2010;20:945-952.

3 Chan JK, Teoh D, Hu JM, et al. Do clear cell ovarian carcinomas have poorer prognosis compared to other epithelial cell types? A study of 1411 clear cell ovarian cancers. Gynecol Oncol 2008;109:370-376.

4 Goff BA, Sainz de la Cuesta R, Muntz HG, et al. Clear cell carcinoma of the ovary: a distinct histologic type with poor prognosis and resistance to platinum-based chemotherapy in stage III disease. Gynecol Oncol 1996;60:412-417.

5 Sugiyama T, Kamura T, Kigawa J, et al. Clinical characteristics of clear cell carcinoma of the ovary: a distinct histologic type with poor prognosis and resistance to platinum-based chemotherapy. Cancer 2000;88:2584-2589.

6 Thomas M, Mariani A, Wright JD, et al. Surgical management and adjuvant therapy for patients with uterine clear cell carcinoma: a multi-institutional review. Gynecol Oncol 2008;108:293-297.

7 Becht E, Giraldo NA, Germain C, et al. Immune contexture, immunoscore, and malignant cell molecular subgroups for prognostic and theranostic classifications of cancers. Adv Immunol 2016;130:95-190.

8 Hanahan D, Weinberg RA. Hallmarks of cancer: the next generation. Cell 2011;144:646-674.

9 Iwai Y, Ishida M, Tanaka Y, et al. Involvement of PD-L1 on tumor cells in the escape from host immune system and tumor immunotherapy by PD-L1 blockade. Proc Natl Acad Sci USA 2002;99:12293-12297.

10 Okazaki T, Iwai Y, Honjo T. New regulatory co-receptors: inducible co-stimulator and PD-1. Curr Opin Immunol 2002;14:779-782.

11 Park JJ, Omiya R, Matsumura Y, et al. B7-H1/CD80 interaction is required for the induction and maintenance of peripheral T-cell tolerance. Blood 2010;116: 1291-1298.

12 Butte MJ, Keir ME, Phamduy TB, et al. Programmed death-1 ligand 1 interacts specifically with the B7-1 costimulatory molecule to inhibit $\mathrm{T}$ cell responses. Immunity 2007;27:111-122.

13 Dong H, Strome SE, Salomao DR, et al. Tumorassociated B7-H1 promotes T-cell apoptosis: a potential mechanism of immune evasion. Nat Med 2002;8: 793-800.

14 Reiss KA, Forde PM, Brahmer JR. Harnessing the power of the immune system via blockade of PD-1 and PD-L1: a promising new anticancer strategy. Immunotherapy 2014;6:459-475.

15 Taube JM, Klein A, Brahmer JR, et al. Association of PD-1, PD-1 ligands, and other features of the tumor immune microenvironment with response to anti-PD-1 therapy. Clin Cancer Res 2014;20:5064-5074.

16 Zou W, Chen L. Inhibitory B7-family molecules in the tumour microenvironment. Nat Rev Immunol 2008;8: 467-477.

17 Brahmer J, Reckamp KL, Baas P, et al. Nivolumab versus docetaxel in advanced squamous-cell non-small-cell lung cancer. N Engl J Med 2015;373:123-135.

18 Reck M, Rodriguez-Abreu D, Robinson AG, et al. Pembrolizumab versus chemotherapy for PD-L1positive non-small-cell lung cancer. N Engl J Med 2016;375:1823-1833.

19 Howitt BE, Shukla SA, Sholl LM, et al. Association of polymerase e-mutated and microsatellite-instable endometrial cancers with neoantigen load, number of tumor-infiltrating lymphocytes, and expression of PD-1 and PD-L1. JAMA Oncol 2015;1:1319-1323.

20 Sloan EA, Ring KL, Willis BC, et al. PD-L1 expression in mismatch repair-deficient endometrial carcinomas, including lynch syndrome-associated and MLH1 promoter hypermethylated tumors. Am J Surg Pathol 2017;41:326-333.

21 Cree IA, Booton R, Cane P, et al. PD-L1 testing for lung cancer in the UK: recognizing the challenges for implementation. Histopathology 2016;69:177-186.

22 Brahmer JR, Tykodi SS, Chow LQ, et al. Safety and activity of anti-PD-L1 antibody in patients with advanced cancer. N Engl J Med 2012;366:2455-2465.

23 Gadgeel SM, Stevenson J, Langer C, et al. Pembrolizumab (pembro) plus chemotherapy as front-line therapy 
for advanced NSCLC: KEYNOTE-021 cohorts A-C. J Clin Oncol 2016;34(Suppl): abstr 9016.

24 Bristol-myers squibb announces top-line results from CheckMate -026, a phase 3 study of opdivo (nivolumab) in treatment-naïve patients with advanced non-small cell lung cancer Opdivo did not meet trial primary endpoint of progression-free survival in patients expressing PD-L1 $\geq 5 \%$. Available at http://news.bms. $\mathrm{com} /$ press-release/bristolmyers/bristol-myers-squibbannounces-top-line-results-checkmate-026-phase-3stu. Updated 5 August 2016. Accessed 26 August 2016.

25 Cimino-Mathews A, Thompson E, Taube JM, et al. PD-L1 (B7-H1) expression and the immune tumor microenvironment in primary and metastatic breast carcinomas. Hum Pathol 2016;47:52-63.

26 Thompson E, Taube JM, Elwood H, et al. The immune microenvironment of breast ductal carcinoma in situ. Mod Pathol 2016;29:249-258.

27 Engel JB, Honig A, Kapp M, et al. Mechanisms of tumor immune escape in triple-negative breast cancers (TNBC) with and without mutated BRCA 1. Arch Gynecol Obstet 2014;289:141-147.

28 Joneja U, Vranic S, Swensen J, et al. Comprehensive profiling of metaplastic breast carcinomas reveals frequent overexpression of programmed deathligand 1. J Clin Pathol 2017;70:255-259.

29 Dill EA, Gru AA, Atkins KA, et al. PD-L1 expression and intratumoral heterogeneity across breast cancer subtypes and stages: an assessment of 245 primary and 40 metastatic tumors. Am J Surg Pathol 2017;41: 334-342.

30 Friedman K, Brodsky AS, Lu S, et al. Medullary carcinoma of the colon: a distinct morphology reveals a distinctive immunoregulatory microenvironment. Mod Pathol 2016;29:528-541.

31 Diaz LA Jr, Le DT. PD-1 blockade in tumors with mismatch-repair deficiency. N Engl J Med 2015;373: 1979.

32 Webb JR, Milne K, Kroeger DR, et al. PD-L1 expression is associated with tumor-infiltrating $\mathrm{T}$ cells and favorable prognosis in high-grade serous ovarian cancer. Gynecol Oncol 2016;141:293-302.

33 Gottlieb CE, Mills AM, Cross JV, et al. Tumorassociated macrophage expression of PD-L1 in implants of high grade serous ovarian carcinoma: a comparison of matched primary and metastatic tumors. Gynecol Oncol 2017;144:607-612.

34 Strickland KC, Howitt BE, Shukla SA, et al. Association and prognostic significance of BRCA1/2-mutation status with neoantigen load, number of tumorinfiltrating lymphocytes and expression of PD-1/PDL1 in high grade serous ovarian cancer. Oncotarget 2016;7:13587-13598.

35 Ketabi Z, Bartuma K, Bernstein I, et al. Ovarian cancer linked to lynch syndrome typically presents as earlyonset, non-serous epithelial tumors. Gynecol Oncol 2011;121:462-465.

36 Cai KQ, Albarracin C, Rosen D, et al. Microsatellite instability and alteration of the expression of hMLH1 and hMSH2 in ovarian clear cell carcinoma. Hum Pathol 2004;35:552-559.

37 Strickland KC, Howitt BE, Rodig SJ, et al. Immunogenicity of clear cell ovarian cancer: association with ARID1A loss, microsatellite instability and endometriosis. J Clin Oncol 2016;34(Suppl): abstr 5514.

38 Herzog T, Arguello D, Reddy S, et al. PD-1 and PD-L1 expression in 1599 gynecological malignancies - implications for immunotherapy. Gynecol Oncol 2015;137 (Suppl): 1.

39 Stewart CJ, Bowtell DD, Doherty DA, et al. Long-term survival of patients with mismatch repair proteindeficient, high-stage ovarian clear cell carcinoma. Histopathology 2017;70:309-313.

40 Folkins AK, Longacre TA. Hereditary gynaecological malignancies: advances in screening and treatment. Histopathology 2013;62:2-30.

41 Sloan EA, Moskaluk CA, Mills AM. Mucinous differentiation with tumor infiltrating lymphocytes is a feature of sporadically methylated endometrial carcinomas. Int J Gynecol Pathol 2016 [e-pub ahead of print 10 August 2016].

42 Asaoka Y, Ijichi H, Koike K. PD-1 blockade in tumors with mismatch-repair deficiency. $N$ Engl J Med 2015;373:1979.

43 Wiegand KC, Shah SP, Al-Agha OM, et al. ARID1A mutations in endometriosis-associated ovarian carcinomas. N Engl J Med 2010;363:1532-1543.

44 Pearce CL, Templeman C, Rossing MA, et al. Association between endometriosis and risk of histological subtypes of ovarian cancer: a pooled analysis of casecontrol studies. Lancet Oncol 2012;13:385-394.

45 Zorn KK, Bonome T, Gangi L, et al. Gene expression profiles of serous, endometrioid, and clear cell subtypes of ovarian and endometrial cancer. Clin Cancer Res 2005;11:6422-6430.

46 Fadare O, Zheng W, Crispens MA, et al. Morphologic and other clinicopathologic features of endometrial clear cell carcinoma: a comprehensive analysis of 50 rigorously classified cases. Am J Cancer Res 2013;3: 70-95.

47 Jones S, Wang TL, Shih I, et al. Frequent mutations of chromatin remodeling gene ARID1A in ovarian clear cell carcinoma. Science 2010;330:228-231.

48 Fata CR, Seeley EH, Desouki MM, et al. Are clear cell carcinomas of the ovary and endometrium phenotypically identical? A proteomic analysis. Hum Pathol 2015;46:1427-1436.

49 Fadera AN, Diazb LA, Armstrong DK, et al. Preliminary results of a phase II study: PD-1 blockade in mismatch repair-deficient, recurrent or persistent endometrial cancer. Abstracts Presented for the 47th Annual Meeting of the Society of Gynecologic Oncology Scientific. Gynecol Oncol 2016;141:206-207.

50 Ott PA, Bang Y, Berton-Rigaud D, et al. Pembrolizumab in advanced endometrial cancer: preliminary results from the phase ib KEYNOTE-028 study. J Clin Oncol 2016;34(Suppl): abstr 5581.

51 Santin AD, Bellone S, Buza N, et al. Regression of chemotherapy-resistant polymerase epsilon (POLE) ultra-mutated and MSH6 hyper-mutated endometrial tumors with nivolumab. Clin Cancer Res 2016;22: 5682-5687.

52 Hamanishi J, Mandai M, Konishi I. Immune checkpoint inhibition in ovarian cancer. Int Immunol 2016;28: 339-348.

53 Hamanishi J, Mandai M, Ikeda T, et al. Safety and antitumor activity of anti-PD-1 antibody, nivolumab, in patients with platinum-resistant ovarian cancer. J Clin Oncol 2015;33:4015-4022.

54 Disis ML, Patel MR, Pant S, et al. Avelumab (MSB0010718C), an anti-PD-L1 antibody, in patients with previously treated, recurrent or refractory ovarian cancer: a phase Ib, open-label expansion trial. J Clin Oncol 2015;33(Suppl): abstr 5509. 
55 Bennett JA, Morales-Oyarvide V, Campbell S, et al. Mismatch repair protein expression in clear cell carcinoma of the ovary: Incidence and morphologic associations in 109 cases. Am J Surg Pathol 2016;40: 656-663.

56 Vierkoetter KR, Ayabe AR, VanDrunen M, et al. Lynch syndrome in patients with clear cell and endometrioid cancers of the ovary. Gynecol Oncol 2014;135:81-84.

57 Topalian SL, Hodi FS, Brahmer JR, et al. Safety, activity, and immune correlates of anti-PD-1 antibody in cancer. N Engl J Med 2012;366:2443-2454.

58 Robert C, Long GV, Brady B, et al. Nivolumab in previously untreated melanoma without BRAF mutation. N Engl J Med 2015;372:320-330.

59 Powles T, Eder JP, Fine GD, et al. MPDL3280A (antiPD-L1) treatment leads to clinical activity in metastatic bladder cancer. Nature 2014;515:558-562.
60 Larkin J, Chiarion-Sileni V, Gonzalez R, et al. Combined nivolumab and ipilimumab or monotherapy in untreated melanoma. N Engl J Med 2015;373:23-34.

61 Scheel AH, Dietel M, Heukamp LC, et al. Harmonized PD-L1 immunohistochemistry for pulmonary squamous-cell and adenocarcinomas. Mod Pathol 2016;29: 1165-1172.

62 Mahoney KM, Sun H, Liao X, et al. PD-L1 antibodies to its cytoplasmic domain most clearly delineate cell membranes in immunohistochemical staining of tumor cells. Cancer Immunol Res 2015;3:1308-1315.

63 Heinzerling L, Goldinger SM. A review of serious adverse effects under treatment with checkpoint inhibitors. Curr Opin Oncol 2017;29:136-144.

64 Hughes PE, Caenepeel S, Wu LC. Targeted therapy and checkpoint immunotherapy combinations for the treatment of cancer. Trends Immunol 2016;37:462-476. 